Lexis Vol. XLI (1) 2017: 203-230

\title{
Influencia del español en el sistema vocálico shuar: vocales susurradas
}

\author{
Miroslav Valeš \\ Universidad Técnica de Liberec, República Checa
}

\section{RESUMEN}

El objetivo del siguiente artículo es presentar un estudio sobre las vocales susurradas, y su gradual desaparición, en la lengua shuar. El estudio parte de la premisa de que las vocales susurradas desaparecen de la lengua shuar por la acomodación al sistema vocálico del español. Este estudio se fundamenta en la investigación realizada en 21 comunidades a lo largo del territorio shuar. Se examinan tres factores extralingüísticos: edad, localidad y grado del bilingüismo. La conclusión confirma que el contacto con el español juega un papel importante en la desaparición de las vocales susurradas porque la intensidad del contacto es el factor que más influye en su desuso gradual.

Palabras clave: shuar, vocales, susurradas, edad, localidad

\section{AbSTRACT}

The objective of the following paper is to present a study about the voiceless vowels in Shuar and their decreasing vitality. The hypothesis of the study presumes that the voiceless vowels disappear due to the influence of Spanish vocalic system. The database for the research was collected in 21 Shuar communities. The study takes into account three extralinguistic factors: age, locality and grade of bilingualism. The conclusion confirms that the contact with Spanish plays an important role in the disappearance

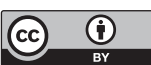


of the voiceless vowels as the intensity of contact with Spanish is the most influential factor in their gradual disuse.

Keywords: Shuar, voiceless, vowels, age, locality

\section{Introducción}

La lengua shuar - igual que otras lenguas indígenas de Ecuadorestá en contacto intensivo con español. Aunque la Constitución vigente del año 2008 proclama que el shuar es la lengua “oficial de relación intercultural” y asegura que "[e]l Estado respetará y estimulará su conservación y uso" (Constitución de la República del Ecuador: art. 2), la situación de contacto lingüístico favorece indudablemente al español como una lengua de gran dominio. El español está visto como una lengua que potencia el avance económico, mientras que el shuar está relacionado con el pasado, con las tradiciones y el folclore. A causa de estas condiciones favorables para el español, avanza el bilingüismo inestable y las generaciones jóvenes pasan gradualmente al monolingüismo en la lengua española (Valeš 2011).

Por lo visto, el contacto lingüístico no tiene su implicación solo en los avances de las tasas de bilingüismo y monolingüismo en español, sino también en la acomodación del sistema fonéticofonológico propio de la lengua shuar a la estructura del sistema ajeno. El objetivo del presente trabajo será el de analizar cómo se refleja esta situación de contacto en el gradual desuso de las vocales susurradas, sonidos que solo existen en muy pocas lenguas del mundo ${ }^{1}$. El estudio parte de la premisa de que las vocales susurradas desaparecen de la lengua shuar por la influencia del sistema vocálico español. En la primera parte del trabajo, se exponen las cualidades fonéticas, las dudas respecto a la relevancia fonológica de las vocales susurradas y su vitalidad. En la segunda

1 Otra de las lenguas que tiene vocales susurradas en su inventario es awapit, lengua hablada por la etnia Awa en el noroeste de Ecuador (Henriksen y Obando 1985: viii). 
parte, se discuten los problemas metodológicos y las limitaciones dadas por la metodología utilizada y la tercera parte examina tres factores extralingüísticos: edad, localidad y grado del bilingüismo, y su influencia en la conservación o desaparición de las vocales susurradas.

Según se ha comprobado en varias ocasiones, los sistemas lingüísticos evolucionan de manera acelerada cuando se encuentran bajo presión social (Labov 1972: 178). La situación de la lengua shuar es parecida a la situación de muchas lenguas indígenas del continente Americano. La lengua vernácula sirve como medio de comunicación familiar, mientras que la lengua dominante — el español en este caso- sirve como vehículo de la educación y comunicación fuera del ambiente familiar o comunitario. Consecuentemente, la lengua dominante está vista como un idioma que tiene más potencial económico y ofrece más oportunidades. Esta situación de estatus desigual tiene luego inevitablemente una repercusión en el sistema lingüístico mismo.

Queda demostrado en varios estudios (p. ej. Siguán 2001: 176177) que las interferencias ocurren en todos los niveles de la lengua: fonético, prosódico, léxico, semántico y morfosintáctico. Los cambios más llamativos se manifiestan en la presencia del léxico de la lengua dominante en la lengua minoritaria (Valeš 2005); sin embargo, los cambios se pueden observar también en otros niveles. Los sistemas fonológicos de dos lenguas pertenecientes a diferentes familias suelen ser divergentes y, por esta razón, no es sorprendente que las personas bilingües tiendan a superponer uno sobre el otro. El ajuste del sistema fonético en los ambientes bilingües aporta a los usuarios la ventaja de no tener que cambiar el sistema siempre que se cambia el contexto del habla. Por esta razón, el sistema fonético de la lengua dominante suele proyectar sus puntos y modos de articulación al sistema de la lengua minoritaria. Estos cambios ocurren de manera gradual. Cuando se hacen frecuentes las interferencias fonéticas, se pueden convertir en una norma y cambiar o precipitar el cambio del sistema fonológico. Sin embargo, como advierte Matras (2009), existen también influencias que impiden este tipo de cambios: 
From the point of view of handling the multilingual repertoire, there is a functional motivation favouring consistency in the types and points of articulation as well as the distribution rules of allophonic variation and suprasegmentals, regardless of the speech situation in which language users find themselves. This motivation exerts pressure toward convergence of the two phonological 'systems' in the speaker's repertoire. At the same time, social norms, awareness of identity, and loyalty toward the group associated with the home language may counteract levelling within the phonological repertoire by demanding conformity to the established pronunciation norms (225).

Los resultados referentes al shuar, que se exponen a continuación, describen la influencia del sistema vocálico español en la evolución acelerada del shuar. En este caso particular, se trata de la eliminación completa de los elementos ausentes en el sistema vocálico español.

\section{Sistema vocálico shuar y las vocales susurradas}

La lengua shuar pertenece a la familia lingüística que se denomina jíbaro pero creemos que la terminología más adecuada sería familia lingüística "chicham" para evitar el término despectivo. A la familia chicham pertenecen las lenguas: shuar -en el sur-este de Ecuador-, achuar (achuar-shiwiar) -en el este de Ecuador y norte del Perú-, wampís (huambisa) -en Perú, a lo largo de los ríos Santiago y Morona- y awajún (aguaruna) -en la Amazonia peruana de las provincias Loreto y Amazonas-.

En general, la información disponible sobre la fonética y fonología shuar es insuficiente y bastante anticuada. Basándose en la descripción de Turner (1958a: 1992) y Gnerre (1999: 2010) el sistema vocálico de la lengua shuar cuenta con 12 fonemas.

Tabla 1. Sistema vocálico shuar (según Turner 1992: 14)

\begin{tabular}{cccc}
\hline vocal & anterior & central & posterior \\
\hline cerrada & $\mathrm{i} \tilde{\mathrm{i}}^{\mathrm{i}}$ & $\mathrm{i}^{\tilde{\mathrm{t}}^{\mathrm{i}}}$ & $\mathrm{u} \tilde{\mathrm{u}}^{\mathrm{u}}$ \\
media & & & \\
abierta & & $\mathrm{a} \tilde{\mathrm{a}}^{\mathrm{a}}$ & \\
\hline
\end{tabular}


Según Turner (1992: 14), los cuatro fonemas shuar básicos /i, i, a, $\mathrm{u} /$ están complementados por juegos iguales de fonemas nasales:

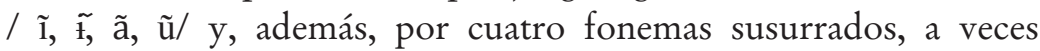
denominados sordos: $/ \mathrm{i}, \mathrm{i}, \mathrm{a}, \mathrm{u} /$. Sin embargo, nuestro estudio no confirma esta premisa, ya que no encontramos razones suficientes para incluir las vocales susurradas entre los fonemas. En otro estudio del autor (Valeš, in review) se discute también el carácter fonémico cuestionable de las vocales nasales. En shuar existen, además, las vocales largas, por ejemplo, en las palabras heencham (murciélago), unnt (grande), paat (caña), etcétera: pero a diferencia de las susurradas y las nasales, las vocales largas nunca han sido consideradas fonemas.

Tabla 2. Sistema vocálico español

\begin{tabular}{|c|c|c|c|}
\hline vocal & anterior & central & posterior \\
\hline cerrada & $\mathrm{i}$ & & $\mathrm{u}$ \\
\hline media & $\mathrm{e}$ & & o \\
\hline abierta & & $\mathrm{a}$ & \\
\hline
\end{tabular}

Si comparamos el sistema shuar con el del español, expuesto en la tabla 2, notamos diferencias sustanciales. Los fonemas /i, a, u/ corresponden aproximadamente a sus equivalentes españoles. El fonema / $\mathbf{i}$ / es una vocal central cerrada que no tiene equivalencia en el sistema vocálico español. Su representación gráfica es “e”. Las vocales de apertura media [e, o] no están ausentes en shuar, pero no tienen el carácter fonológico. El sonido [e] es alófono de la /a/ según argumenta Turner (1958b: 87-89). Este sonido aparece con frecuencia, por ejemplo, entre la consonante $/ \mathrm{h} /$ y la vocal /i/: winiajai [wináhei] (vengo). El sonido [o] es también alófono de la /a/ que aparece antes o después de la /u/: yajauch [jax óut'] (malo). Según Turner (1992: 16-19), el tercer alófono de la /a/ es un sonido abierto [æ] que aparece entre /i/ y /r/: kiárai [kiærei] (tarde).

Los fonemas vocálicos nasales no tienen sus paralelos en el sistema fonológico español, sin embargo, las vocales nasales existen en el español con estatus de alófonos. Según afirma Gnerre (2010), "[l]a nasalización ha debido tener en el pasado una función bastante 
significativa en las lenguas jíbaras” (47). No obstante, exponiendo la variación libre en algunos contextos, admite (Gnerre) que actualmente "el rendimiento funcional de las vocales nasales es muy bajo" (48).

Las vocales susurradas, sujeto de este estudio, solo aparecen al final de la palabra/enunciado, suenan como una aspiración y algunos autores (p. ej. Fast Mowitz 1975a, 1975b) las llaman vocales sordas. Gnerre (2010) escribe al respecto: "Una cuarta característica importante de las vocales es la de su ejecución susurrada, de rara presencia en el habla espontánea y, en todo caso, presente solo en la posición final del enunciado" (47). Si una palabra terminada en una vocal susurrada, por ejemplo [ipják ${ }^{u}$ (achiote), aparece en la cadena hablada, esta vocal se pronuncia como una vocal plena: [ipjákuitij] (es achiote). Por esta limitación respecto a su posición las vocales susurradas no son de uso frecuente en el habla espontánea. Gnerre (2010) también expone una regla de "desonorización de vocal oral final de palabra en morfemas léxicos tri- o tetravocálicos en nombre o adjetivo”, pero admite, que "[e]sta regla solo la aplican algunos hablantes (quizás los más ancianos) siempre en estilo de citación, pero no en la conversación rápida. En este caso o en el estilo "negligente", se produce una supresión o elisión de la vocal final de la palabra” (64).

Las cualidades acústicas de las vocales susurradas las podemos observar en los espectrogramas 1 y 2 . En estos notamos, sobre todo, la baja amplitud de estas vocales, y la falta de los formantes. En comparación con las vocales orales, las cuerdas vocales no están vibrando y solo aparece ruido.

Otro aspecto que notamos al estudiar las vocales susurradas es su poca relevancia fonológica. Pares de palabras en las cuales las vocales susurradas diferencian el significado, por ejemplo, [ká:pỉ] (mosca), [ká:pi] (bejuco) son muy escasos, de hecho este ejemplo es el único que conseguimos encontrar. Eso también significa que para la /u/ y la $/$ a / susurradas no existen pares donde distinguen significado $y$, por lo tanto, son fonemas cuestionables. Además, en caso de la vocal /a/ el diccionario Chicham (Pellizzaro y Náwech 2005) solo recoge dos palabras donde aparece esta vocal: múuk ${ }^{a}$ (cabeza) y mutsuk ${ }^{a}$ (cerebro). Tampoco encontramos pares mínimos en los que la susurrada distinguiera el significado en confrontación con una vocal oral. Por estas 
razones, parece que las cuatro vocales susurradas a veces se consideran fonemas más bien por tradición (Turner 1958a, 1992; Gnerre 2010) y para guardar la simetría del sistema, que por razones puramente fonológicas. A pesar de que se trata, en realidad, de un fenómeno fonético, más que fonológico, las vocales susurradas forman parte del sistema shuar que se encuentra en el proceso de cambio.

Espectrograma 1: kaap ${ }^{i}$ (bejuco)

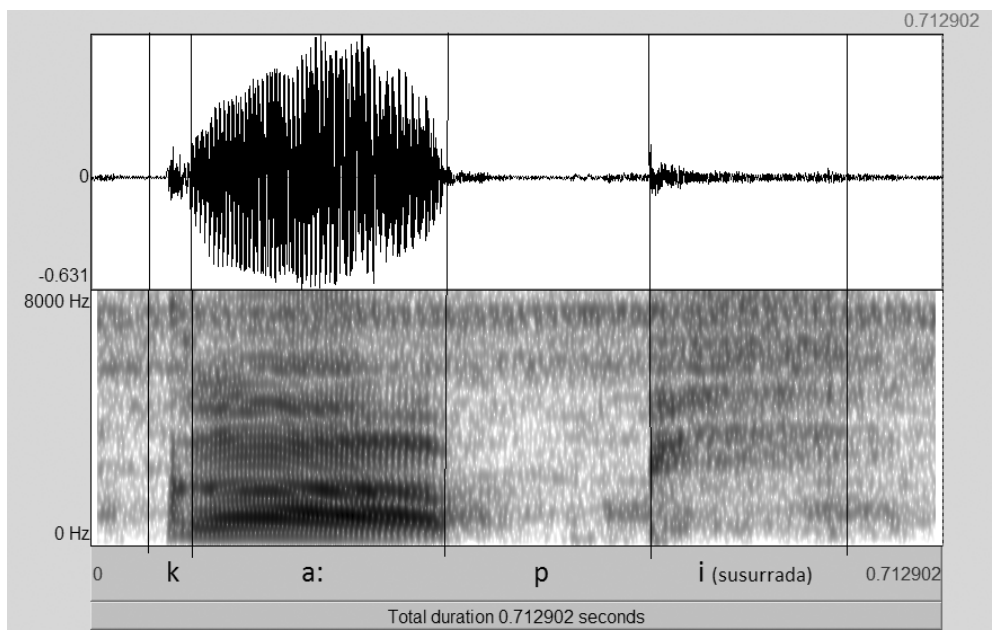

Espectrograma 2: ipiak ${ }^{u}$ (achiote)

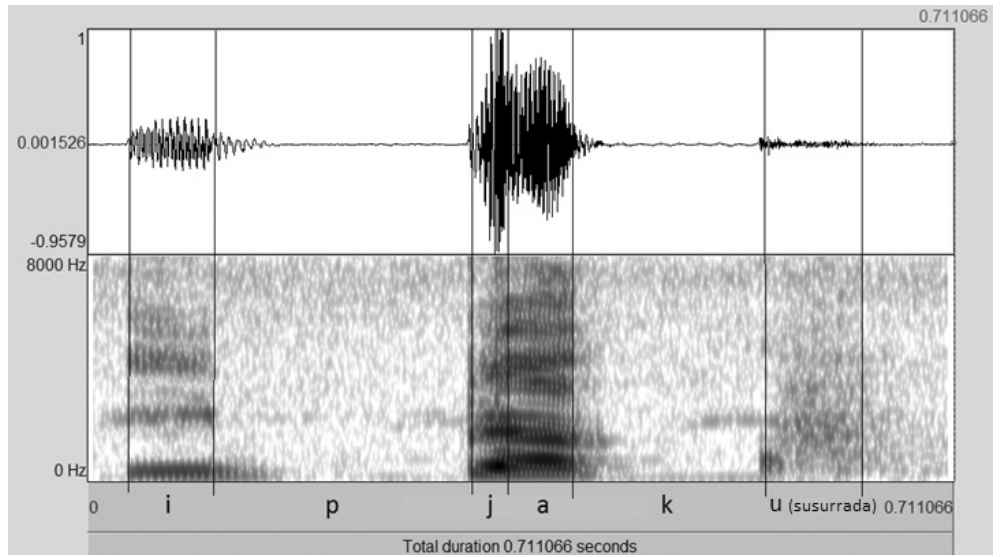


En los diccionarios aparece también el diptongo susurrado [iu], sin embargo, este se encuentra siempre después de una consonante palatalizada, por ejemplo, menáint ${ }^{\text {iu }}$ (tres), iwiáink ${ }^{i u}$ (árbol venenoso), katsuint ${ }^{i n}$ (planta, variedad más grande de tsapa), kawitiu (árbol con cuya savia se ablanda el cabello), y por esa razón se trata más bien de palatalización seguida por la vocal susurrada /u/.

Respecto a la vitalidad de las vocales susurradas, es indudable que su uso es cada vez menos frecuente. Gnerre (2010) afirma que las vocales susurradas las utilizan "solo algunos hablantes (quizás los más ancianos)” (64). También en las otras lenguas de la misma familia se ha observado la pérdida de las vocales susurradas. Fast Mowitz (1975b), refiriéndose al achuar, comenta que: “Generalmente las vocales sordas se han perdido completamente en el habla de algunos hablantes del idioma, mientras que en el habla de otros existe una fluctuación entre vocales sordas y sonoras o se pierden por completo" (14). De igual forma, en el Diccionario Aguaruna (Wipio Deicat 1996) se sostiene que "[1]as vocales entre paréntesis son sordas. Estas vocales se están perdiendo poco a poco, pero en algunas áreas todavía se pronuncian" (16). Estas afirmaciones sugieren que el proceso de la pérdida de las vocales susurradas empezó ya hace muchos años y no avanza igual de rápido en toda la comunidad de habla. Como el proceso de la pérdida afecta a todas las lenguas de la familia se podría suponer que se trata del proceso interno de las lenguas, sin embargo, todas las lenguas de esta familia se encuentran en contacto con el español y, por lo tanto, la evolución acelerada también se puede deber a la presión social ocasionada por el contacto lingüístico.

La pérdida gradual se puede observar también si comparamos los diccionarios de fecha más antigua con los publicados recientemente. El diccionario del Padre Luis Bolla (Bolla 1972), que a su vez incluye el texto completo del Vocabulario del Padre Juan Ghinassi del 1939, contiene muchas palabras con vocales susurradas. En primer lugar, llama la atención la cantidad de verbos que contienen una vocal susurrada. Por ejemplo, ai-k $k^{i}$ (desnudar), aki-ki (pagar), jikiat (amar de corazón), etcétera. Esta circunstancia parece inusual, ya que los verbos casi siempre aparecen con algún sufijo que los determina 
gramaticalmente, o sea, que es difícil encontrar un contexto donde el verbo aparezca sin sufijo y con la vocal final susurrada. En segundo lugar, este diccionario pone en tela de juicio la premisa de que las vocales susurradas solo aparecen al final del enunciado. El diccionario contiene una cantidad de términos compuestos de dos palabras donde la vocal susurrada aparece en la primera sin finalizar el enunciado. Por ejemplo, jimpikit ${ }^{i}$ chinki (ave roja), kunkuk ${ }^{i}$ napi (serpiente de los árboles), sumpit ${ }^{i}$ atash (gallina corta), etcétera. Por esta razón será más prudente considerar que las vocales susurradas aparecen al final de palabra, pero no siempre tienen que finalizar el enunciado. La última, más importante, y ya mencionada observación de este diccionario es la frecuencia de las vocales susurradas. Estas aparecen en las palabras donde no las registran los diccionarios posteriores. Por ejemplo, pinink ${ }^{i}$ (plato, pilche de barro cocido), akájik ${ }^{i}$ (trenzas de cabello), etc. A base de estas observaciones es probable que hace $50 \mathrm{o}$ más años la frecuencia de las vocales susurradas fuera superior.

El diccionario Chicham (Pellizzaro y Náwech 2005) publicado más recientemente registra menos vocales susurradas en comparación con el anterior; aun así, estas aparecen en palabras en las que no las pronunció ninguno de los participantes de este estudio. Se trata, por ejemplo, de las palabras namank $k^{e}$ (carne) y eweje (cinco), donde todos los participantes pronunciaron solo la consonante final. Parece que el proceso de la desaparición de las vocales susurradas sigue avanzando, y quedan efectivamente pocas palabras donde los hablantes utilizan este sonido que no tiene su paralelo en el sistema fonético del español. El tercer diccionario examinado: Chicham nekatai (SERBISH 2004) no marca las vocales susurradas en ninguna palabra. Es probable que este diccionario siga la ortografía habitual que no marca estos fonemas. Igual observación hicimos en la traducción de la Constitución de la República del Ecuador a la lengua shuar -Ecuador nunka umíktin umpuarma-. Es evidente que este tipo de ortografía apoya la desaparición de las vocales susurradas de la lengua shuar. Igual que en el caso de la descripción fonético-fonológica, la información sobre el léxico shuar es insuficiente, poco fiable y no refleja la variación de la lengua. 


\section{Metodología, recogida de datos e hipótesis}

Los datos que sirven para el siguiente análisis fueron recogidos en 24 comunidades a lo largo del territorio shuar. La base de datos la forman entrevistas grabadas con 84 participantes, en total unas 34 horas de grabaciones. El análisis considera tres factores extralingüísticos: edad, localidad y grado del bilingüismo, y su influencia en la conservación o desaparición de las vocales susurradas.

Para conseguir los datos relevantes sobre las vocales susurradas se utilizó una entrevista dirigida, en la que las respuestas fueron solicitadas por medio de estímulos en forma de fotos. Aunque se trata de un método que no asegura de ninguna manera el uso natural y espontáneo de lenguaje, hubo varias razones para acudir a este método para la recogida de datos. Primero, las entrevistas dirigidas aseguran —en teoría - conseguir las mismas unidades léxicas en un contexto idéntico, o bien, sin contexto. Desgraciadamente, por muy explícitas que fueran las fotos, no todos los hablantes dieron las respuestas en forma esperada. Por ejemplo, la palabra ipiak ${ }^{u}$, solicitada por medio de la foto de los frutos de achiote (Bixa orellana), normalmente provocaba la respuesta deseada pero algunos hablantes, a lo mejor por falta de buena vista, respondían con la palabra genérica kukuj (flor). Problemas más frecuentes aparecían con las palabras $k a a p^{e}$ (mosca) y kaap (liana), que forman un par mínimo. Con la primera los participantes daban nombres de varios insectos, anchi (tábano), chiáchia (cigarra), manchu (zancudo) o ete (avispa). La segunda palabra presentaba una situación parecida, ya que los shuar reconocen muchos tipos de lianas: sin embargo, en el estudio piloto averiguamos que ese tipo de liana se utiliza para fabricar las changuinas (cestas). Bastaba dar la pista "es para hacer la changuina" y la mayoría de los participantes acertaba con la respuesta deseada. Esta situación refleja la importancia del conocimiento de la cultura material de la etnia estudiada para poder formular bien el estímulo. La segunda razón para utilizar las entrevistas dirigidas era la particularidad de que las vocales susurradas solo aparecen al final de palabra/enunciado, o en la pronunciación de palabras sueltas. El 
uso de las vocales susurradas en un discurso espontáneo es poco frecuente y depende esencialmente del contexto. La entrevista con los participantes contenía también una narración libre de un mito, pero en estas narraciones aparecieron las vocales susurradas de manera excepcional. Esta circunstancia requirió solicitar las palabras sin la cadena hablada. La última razón fue la manejabilidad y comparabilidad de los datos. Era deseable que los participantes pronunciaran o no pronunciaran las vocales susurradas en las mismas palabras y en el mismo contexto, por muy artificial que fuera.

La idea original era observar el uso de las vocales susurradas en tres palabras: ipiak" (achiote), kaapi (liana), kaape (mosca), sin embargo, las vocales susurradas aparecieron también en los numerales: menaint ${ }^{u}$ (tres), ujuk ${ }^{e}$ (seis) y, sorprendentemente, en la palabra chaarip ${ }^{i}$ (rayo, relámpago). Esta última aparece en el diccionario Chicham (Pellizzaro y Náwech, 2005) sin la vocal susurrada, a pesar de esto algunos hablantes optaron por pronunciarla con la /i/ susurrada. De estas palabras la mejor representante era la primera: ipiak ${ }^{u}$, por representar una unidad léxica bastante general, y por ser fácilmente identificada por la mayoría de los participantes. En cambio, el par de palabras kaape - kaap $p^{i}$ raras veces aportó las respuestas esperadas por las razones arriba mencionadas.

Los problemas que acompañaron la recogida de datos se deben sobre todo a la situación política en el territorio shuar en consecuencia de la cual muchas comunidades no dejan entrar a personas ajenas a la misma. A causa de esta complicación no fue posible escoger libremente las comunidades que formaron la red de entrevistas, sino que más bien tuvimos que conformarnos con llevar a cabo la investigación en aquellas que estuvieron dispuestas a acoger a un extranjero; por otra parte, una vez aceptado por la comunidad la colaboración fue muy amistosa. 


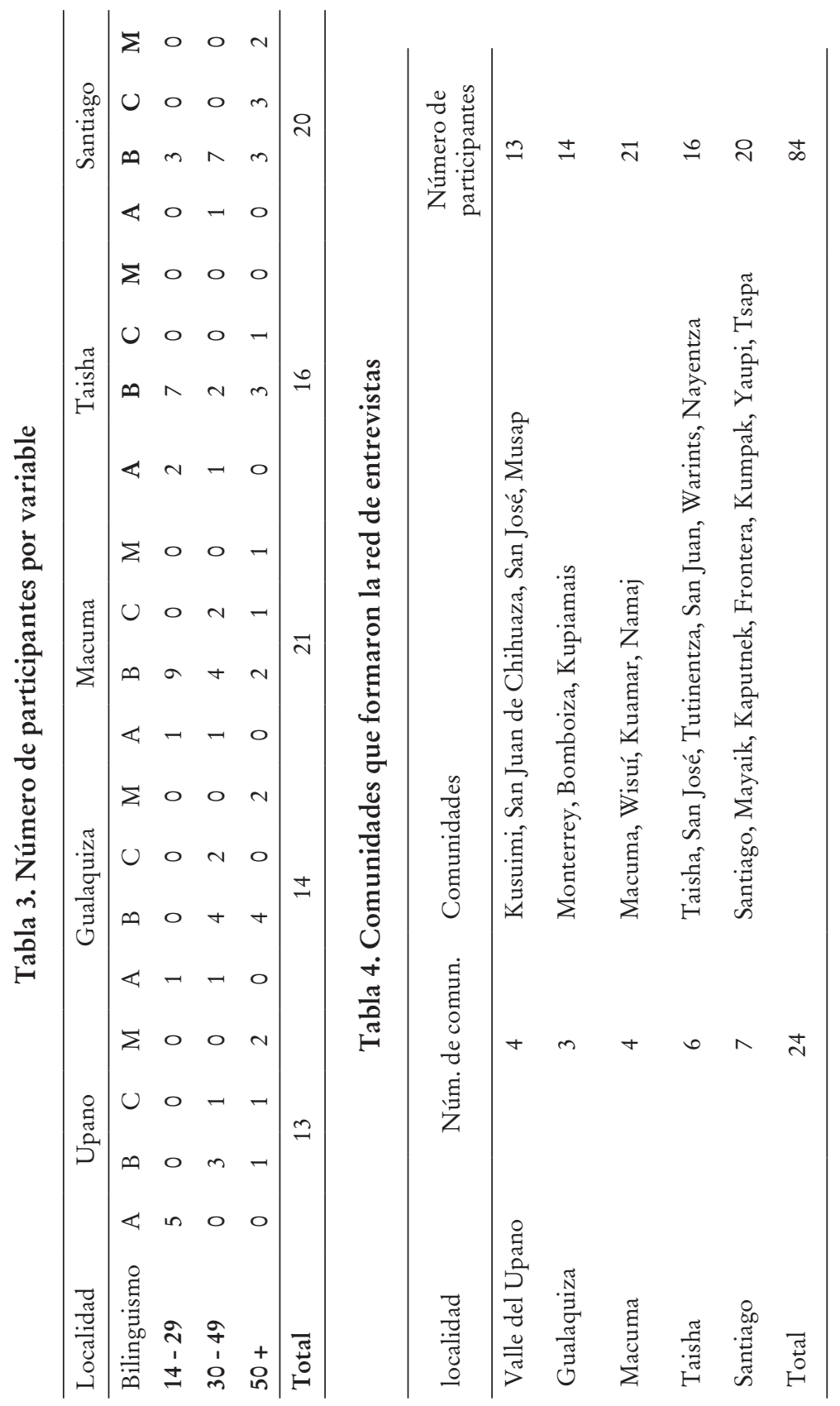


Mapa 1. Comunidades que formaron la red de entrevistas

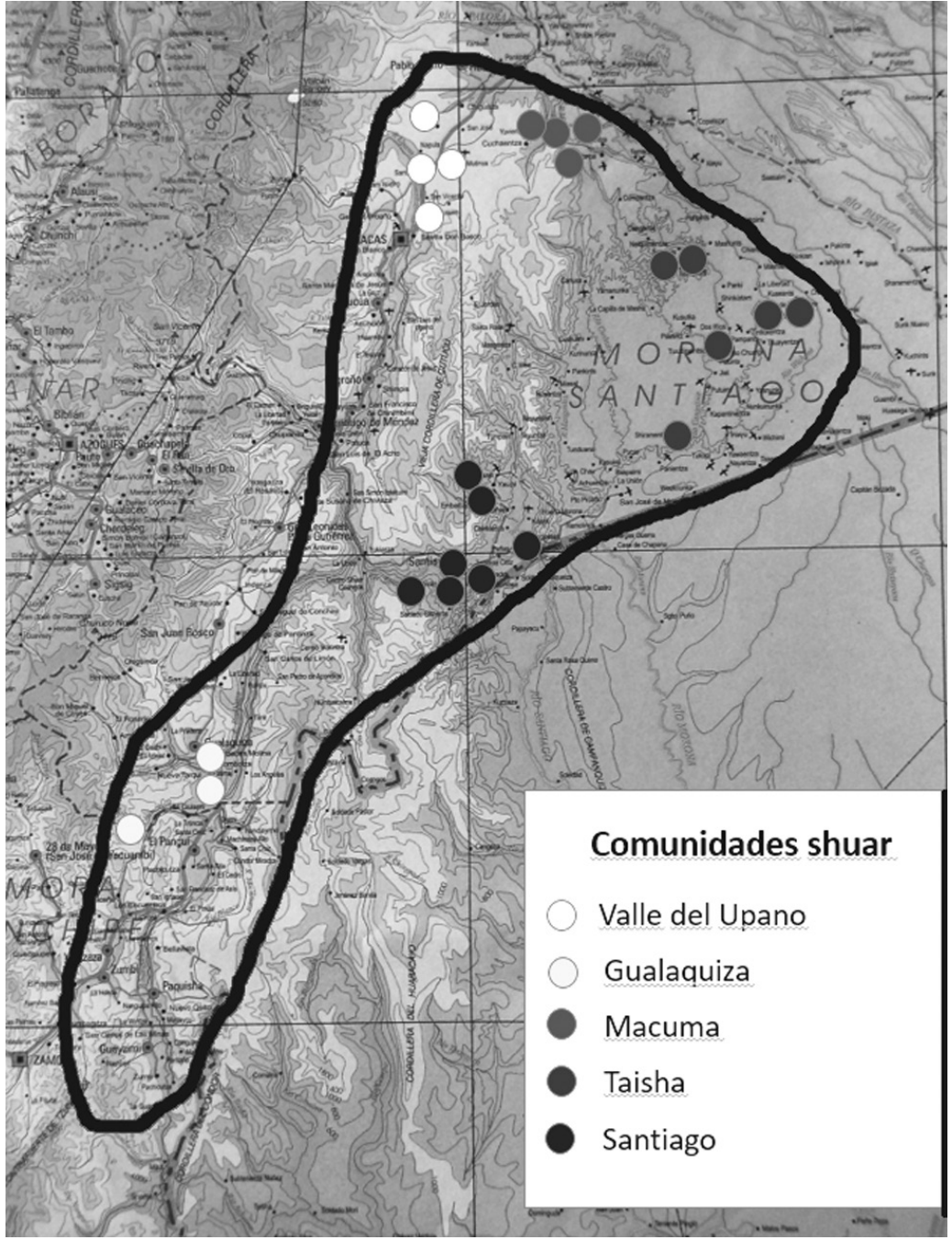

La localidad del participante representa uno de los factores extralingüísticos considerados en el análisis. Para observar la variación diatópica, se intentaban recoger los datos de varias comunidades geográficamente alejadas. Los participantes proceden de 24 comunidades 
diferentes que fueron agrupados para representar cinco áreas geográficas: valle del Upano (norte del territorio shuar), Gualaquiza (sur del territorio shuar), Macuma (parte septentrional del Transcutucú), Taisha (parte oriental del Transcutucú) y Santiago (parte meridional del Transcutucú). Estas cinco áreas geográficas podemos, además, clasificarlas según su historia de colonización. Las primes dos áreas, valle del Upano y Gualaquiza, tienen una historia más larga e intensiva respecto al contacto con la cultura mestiza. Los primeros contactos tras la conquista ocurrieron en el valle del Upano, gracias a los hallazgos de oro en esta área. Sin embargo, después de la sublevación de los shuar contra los españoles en 1599 estos contactos fueron interrumpidos por más de dos siglos. Los contactos se limitaron a las áreas fronterizas y unos pocos puntos de intercambio, por ejemplo, la ciudad de Macas. La historia moderna de los shuar está estrechamente ligada con las misiones de los salesianos. Los primeros salesianos se establecieron en el territorio de los shuar, en Gualaquiza, en 1894. Los contactos y las influencias mutuas están descritas en varias publicaciones (Bottasso 1982, 1993, 2003, 2011; Gnerre 2012; Broseghini 1983). Por esta razón, el valle del Upano y Gualaguiza llevan ya más de un siglo en contacto con la cultura mestiza. En cambio, las tres áreas de Transcutucú están en contacto con la cultura mestiza desde hace poco tiempo. Todavía en la mitad del siglo pasado las áreas detrás de la Cordillera de Cutucú solo eran accesibles a pie o a caballo y los shuar vivían allí de manera tradicional, incluso reduciendo las cabezas de los enemigos asesinados (Hanzelka y Zikmund 1958). El problema relacionado con la variable "localidad" es la alta movilidad de los shuar. En general, los shuar suelen cambiar su lugar de permanencia varias veces en su vida. Por esta razón era difícil buscar personas oriundas del lugar, y algunos de los participantes han vivido en varios lugares durante su vida. Sin embargo, en la mayoría de los casos no se trataba de traslados entre áreas completamente diferentes.

Otros dos factores sociales que se consideran en el análisis son la edad y el grado del bilingüismo. A base de la información accesible (Gnerre 2010: 64) la hipótesis supone que deberían ser las personas mayores las que más utilizan las vocales susurradas. Del mismo modo 
los que dominan mejor el shuar y menos el español deberían utilizar más las formas vernaculares. Esta segunda variable, sin embargo, tiene estrechos lazos con el contacto de la cultura mestiza y, por lo tanto, está parcialmente ligada con las variables "edad" y "localidad". La edad de los participantes en cada comunidad/área geográfica abarca a jóvenes desde 14 años hasta personas muy mayores, personas con escolarización, y sin instrucción formal. Con la excepción de la región Taisha conseguimos encontrar también personas monolingües en la lengua shuar. Con esta amplia gama de participantes se intenta evitar los errores de las descripciones anteriores. Por ejemplo, Karsten $(1935,1989)$ dio descripción de la lengua solo a base de la información de personas mestizas provenientes de Macas. Su trabajo fue después criticado por Harner (1984), quien verificaba los trabajos de Karsten $(1935,1989)$ y Stirling (1938) y sostenía: “I found that there was scarcely a paragraph of ethnographic information in either work that could be considered wholly accurate" (2). Sin embargo, la razón principal para la gran cantidad de informantes es la intención de observar la variación diatópica y diastrática de la lengua shuar, que hasta ahora ha recibido una mínima atención.

La hipótesis de la que parte este trabajo es la variación natural de todas las lenguas y la influencia del sistema fonético de la lengua dominante - en este caso del español— al sistema fonético de la lengua shuar. En concreto, la hipótesis de la investigación se fundamenta en tres puntos de partida. Primero, se esperaba encontrar usos excepcionales de las vocales susurradas que van desapareciendo de la lengua shuar. Segundo, se esperaba encontrar variación diastrática por influencia del factor "edad", suponiendo que las personas mayores van a conservar más las vocales susurradas que los jóvenes. Es cierto que el cambio lingüístico también puede tener su origen en el proceso interno de la lengua. En tal caso la variable edad debería ser la más influyente. Por último, se partió de la hipótesis de que la variación diatópica diferenciaría el valle del Upano y Gualaquiza con las regiones de Transcutucú por el contacto más prolongado e intensivo con la lengua española de las primeras dos. 
4. Vocales susurradas: análisis de datos y resultados

Todas las lenguas vivas varían en el espacio geográfico - variación diatópica-, están sujetas a la variación social — variación diastrática - y se acomodan al estilo del habla — variación diafásicaGracias a esta variación se efectúa el cambio lingüístico y la evolución de las lenguas, que, sin embargo, puede depender también de otros factores. Uno de los factores que ejercen gran influencia es el contacto de lenguas o variedades. Según Weinreich (1979), los efectos del contacto lingǘstico tienen su reflejo en todos los niveles de la lengua. El interés de nuestro análisis será el nivel fonético, en particular el comportamiento de las vocales susurradas, y la influencia de las variables edad, localidad y grado del bilingüismo en dicho comportamiento. Aunque las tres variables se tratarán por separado, se discutirá también la dificultad de separar una de la otra a causa de la evolución natural de la sociedad shuar. En las entrevistas con los 84 participantes documentamos 82 usos de las vocales susurradas y las tablas de resultados exponen los valores absolutos de estos usos.

\subsection{La variable "edad"}

Según la hipótesis, la variable edad, debería ser el factor decisivo en el uso de las vocales susurradas. La información accesible (Gnerre 2010, Fast Mowitz 1975b) que se refería a las vocales susurradas, normalmente, hace referencia a la edad del hablante junto con el gradual desuso de estas vocales.

Para observar la influencia de la variable edad, dividimos a los 84 participantes de la encuesta en tres grupos generacionales. La generación más joven abarca a los participantes entre 14 y 29 años de edad. La generación intermedia la forman los participantes entre 30 y 49 años y la mayor, incluye participantes mayores de 50 años. Los tres grupos están formados por el mismo número de participantes, 28 en cada uno. La segunda columna indica el número total de participantes en la categoría y los que utilizaron una vocal susurrada por lo menos una vez. 
Tabla 5. Resultados de la variable "edad"

\begin{tabular}{|c|c|c|c|c|c|c|c|c|}
\hline \multicolumn{2}{|c|}{$\begin{array}{l}\text { EDAD } \\
\text { part./min. un uso }\end{array}$} & \multirow{2}{*}{$\frac{\text { ká:pi }}{12}$} & \multirow{2}{*}{$\frac{\text { ká:pi }}{2}$} & \multirow{2}{*}{$\frac{\text { ipják }^{u}}{0}$} & \multirow{2}{*}{$\frac{\text { tá: }^{\text {rip }}}{}$} & \multirow{2}{*}{$\frac{\text { minéint }^{\text {ju }}}{0}$} & \multirow{2}{*}{$\frac{\text { ux }^{\mathrm{h} u ́ k}}{0}$} & \multirow{2}{*}{$\begin{array}{c}\text { Total } \\
16\end{array}$} \\
\hline $14-29$ & $28 / 13$ & & & & & & & \\
\hline $30-49$ & 28/17 & 15 & 2 & 3 & 7 & 3 & 0 & 30 \\
\hline $50+$ & $28 / 16$ & 13 & 3 & 12 & 1 & 3 & 4 & 36 \\
\hline
\end{tabular}

Si consideramos los valores absolutos del uso de las vocales susurradas notamos que en la segunda y la tercera generación documentamos casi igual cantidad de usos (30 y 36) mientras que en la generación más joven baja este valor a la mitad (16). A primera vista queda comprobada la hipótesis sobre la influencia de la variable edad, sin embargo, no es solo la generación de los mayores, sino también la segunda generación la que sigue utilizando las vocales susurradas de manera relativamente sistemática. No se verifica entonces la hipótesis de los “más mayores", ya que la segunda generación incluye a los hablantes de más de 30 años. Si además consideramos las personas que utilizaron la vocal susurrada por lo menos una vez, el resultado ya no queda tan convincente. En la primera generación utilizaron este fonema 13 personas, en la segunda 17 y en la tercera 16 participantes. Estas cifras son bastante equilibradas y no marcan de ningún modo la supremacía de la generación más mayor. Se nota que los mayores son más sistemáticos en el uso de las vocales susurradas, las usan con más frecuencia, pero también hay que tener en cuenta la influencia de la variable "grado del bilingüismo”. De los 28 participantes de la generación más joven, 9 utilizaban el español como su lengua principal, siendo capaces de hablar shuar (grado A) y el resto eran personas bilingües (grado B).

En resumen, el uso de las vocales susurradas se documentó con participantes jóvenes, a partir de 16 años de edad. En cifras absolutas, los hablantes de la segunda y tercera generación —a partir de 30 años de edad - utilizan las vocales susurradas con más frecuencia, pero sería equivocado afirmar que son solamente los mayores quienes utilizan este fonema singular. En general, la influencia de 
la variable edad se confirmó solo parcialmente. Es cierto que esta variable ejerce cierta influencia en el uso de las vocales susurradas, pero no queda patente que se trata de la influencia decisiva, ya que se ha documentado sorprendente cantidad de usos de las vocales susurradas entre los hablantes de primera generación y hasta entre los hablantes muy jóvenes.

\subsection{La variable "localidad"}

La variable localidad considera cinco áreas en las que se dividen las 24 comunidades estudiadas. La hipótesis supone encontrar diferencia entre las dos primeras — valle del Upano y Gualaquiza- en comparación con las otras tres áreas restantes. En caso de la variable localidad no conseguimos la misma cantidad de comunidades ni participantes por cada área. Mientras que el número de comunidades oscila entre 3 y 7 por área, la cantidad de participantes fluctúa entre 13 y 21, siendo más representadas Macuma con 21 participantes, y Santiago con 20 participantes. La segunda columna de la tabla señala el número total de participantes en el área y los que utilizaron una vocal susurrada por lo menos una vez.

Tabla 6. Resultados de la variable "localidad"

\begin{tabular}{lcccccccc}
\hline $\begin{array}{l}\text { Localidad } \\
\text { part./min. un uso }\end{array}$ & ká:p $^{\mathrm{i}}$ & ká:p $^{\mathrm{i}}$ & ipják $^{\mathrm{u}}$ & gfá:rip $^{\mathrm{i}}$ & minéint $^{\mathrm{ju}}$ & ux $^{\mathrm{h}}$ úk $^{\mathrm{i}}$ & TOTAL \\
\hline Upano & $13 / 3$ & 1 & 0 & 2 & 2 & 1 & 0 & 6 \\
Gualaquiza & $14 / 3$ & 3 & 0 & 2 & 0 & 1 & 0 & 6 \\
Macuma & $21 / 15$ & 14 & 3 & 4 & 5 & 2 & 1 & 29 \\
Santiago & $20 / 13$ & 11 & 2 & 6 & 2 & 1 & 2 & 24 \\
Taisha & $16 / 12$ & 11 & 2 & 1 & 1 & 1 & 1 & 17 \\
\hline
\end{tabular}

Como se puede notar en la tabla 6 , los resultados de la variable "localidad" confirman plenamente la hipótesis sobre su influencia, tanto si consideramos la cantidad de participantes que usaron una vocal susurrada por lo menos una vez, como si contamos los valores absolutos de usos. El área donde más usos documentamos 
fue Macuma, con 29 usos por 21 participantes, de los cuales 15 utilizaron una vocal susurrada por lo menos una vez. Usos casi igual de frecuentes documentamos también en las otras dos áreas de Transcutucú — Santiago y Taisha-. En cambio, en el valle del Upano y Gualaquiza encontramos solo 6 individuos que utilizaron una vocal susurrada, de los cuales, además, uno era chamán nacido en Taisha con capacidad de comunicación en español limitada. En total, documentamos solo 12 formas en las dos áreas de más contacto con la lengua española.

De los datos disponibles parece que la variable localidad ejerce más influencia que la variable edad. Los hablantes de las localidades más integradas a la cultura mestiza y con más historia de contacto con la lengua española utilizaron las vocales susurradas excepcionalmente, en cambio, los hablantes de las comunidades menos integradas utilizaron estos fonemas con relativa frecuencia. El $70 \%$ de los entrevistados de Transcutucú utilizaron una vocal susurrada por lo menos una vez, mientras que en el valle del Upano y Gualaquiza la cifra llega solo al $22 \%$.

\subsection{La variable "grado del bilingüismo"}

Para observar la variable grado del bilingüismo dividimos los participantes en cuatro grupos según el uso habitual de shuar o español: A, B, C, M. La clasificación se efectuó a base de la observación participante. La investigación siempre suponía una estancia de varios días en cada una de las comunidades estudiadas y, por lo tanto, había suficientes oportunidades para conocer a los entrevistados. La categoría A comprende individuos que son capaces comunicar en shuar pero su lengua habitual de comunicación es español. En la categoría $\mathrm{B}$ incluimos personas plenamente bilingües, que utilizan las dos lenguas dependiendo de la situación, el shuar para la comunicación familiar con personas mayores y español para comunicar con personas ajenas a la comunidad. La categoría C la forman los participantes que son capaces de comunicarse en español, pero lo utilizan excepcionalmente y en su comunicación cotidiana solo utilizan el idioma shuar. La categoría M incluye a los participantes 
monolingües en shuar que no fueron capaces de comunicarse en español. Normalmente se trataba de personas muy mayores, la participante más joven tenía 68 años. La cantidad de participantes por cada categoría es muy desigual, porque la mayoría era plenamente bilingüe. En general, los monolingües (M) y las personas cuya lengua vehicular es shuar $(\mathrm{C})$ son cada vez menos frecuentes. En cambio, la proporción de los que utilizan el español como su lengua de comunicación cotidiana (A) es cada vez más grande. A pesar de que las comunidades con más contacto con el español tienden a tener también más proporción de los hablantes clasificados con el grado A, no todos los que están en esta categoría provenían del valle del Upano y Gualaquiza. La muestra está equilibrada en este respecto y los de bilingüismo A provienen de las cinco áreas estudiadas, igual que los monolingües provienen de todas las localidades excepto de Taisha.

La variable grado del bilingüismo complementa las observaciones de las dos variables anteriores. En cierto modo combina los factores de edad y localidad, ya que los hablantes de la categoría A eran normalmente más jóvenes y provenían de las comunidades más afectadas por la cultura mestiza, o sea, del valle del Upano o de Gualaquiza. En cambio, los participantes de las categorías C y M solían ser más mayores. Como podemos observar, las tres variables no son del todo separables una de otra, a pesar de eso aportan resultados interesantes que se deben interpretar con precaución, considerando el contexto de la recogida de los datos, la estructura y la cantidad limitada de los participantes.

Tabla 7. Resultados de la variable "grado del bilingüismo"

\begin{tabular}{lcccccccc}
\hline $\begin{array}{l}\text { Bilingüismo part./ } \\
\text { min. un uso }\end{array}$ & ká:p $^{\mathrm{i}}$ & ká:p $^{\mathrm{i}}$ & ipják $^{\mathbf{u}}$ & fá:rip $^{\mathrm{i}}$ & minéint $^{\mathrm{ju}}$ & ux $^{\mathrm{h} u ́ k}$ & TOTAL $^{\mathrm{T}}$ \\
\hline A & $13 / 4$ & 2 & 0 & 0 & 2 & 0 & 0 & 4 \\
B & $52 / 31$ & 30 & 6 & 7 & 6 & 4 & 3 & 56 \\
C & $12 / 9$ & 7 & 1 & 6 & 2 & 2 & 1 & 19 \\
M & $7 / 2$ & 1 & 0 & 2 & 0 & 0 & 0 & 3 \\
\hline
\end{tabular}


Los resultados aportan tanto los datos esperados como los menos previsibles. No sorprende que en las entrevistas de los 13 participantes de la categoría A, documentáramos tan solo cuatro vocales susurradas. Estos hablantes acomodan shuar a la lengua de su comunicación habitual —español-, y por lo tanto, no utilizan las vocales susurradas casi nunca. Entre las categorías B y C notamos poca diferencia. Tanto los bilingües como los de lengua habitual shuar utilizaron las vocales susurradas con frecuencia. Aunque los bilingües (B) utilizaron más formas observadas (56), en términos relativos, los hablantes categorizados como $\mathrm{C}$ son los que más vocales susurradas utilizan, 19 formas por 12 hablantes. El resultado más sorprendente lo notamos en la categoría M — los 7 monolingües - , que solo utilizaron tres vocales susurradas. En teoría, estas personas deberían ser las más ligadas a la cultura tradicional shuar y conservar la lengua en su estado original. La explicación de esta anomalía tiene que ver con la metodología de las entrevistas. Como ya hemos mencionado, las personas monolingües eran normalmente muy mayores, algunas ni siquiera sabían cuántos años tenían. Para muchas de estas personas la respuesta a los estímulos en forma de fotos era una tarea demasiado abstracta, o sea, que las respuestas no acertadas eran mucho más frecuentes que en otras categorías observadas. Además, por problemas comunicativos era más difícil explicar a estos participantes que la respuesta deseada comprendía la palabra sin ningún sufijo. Como la gente se expresaba de manera natural, muchas veces recibimos la respuesta en forma: ipiakuiti, que contiene la palabra ipiak + la vocal $u$ pronunciada de manera plena + verbalizador $i t+$ sufijo de tercera persona singular $i$, que en total significa: “es achiote”. En el capítulo 2 explicamos que las vocales susurradas aparecen solo a final de palabra o enunciado y cuando se añade algún sufijo estas vocales se realizan de manera plena. Las respuestas de las personas monolingües naturalmente correspondían con la situación comunicativa pero no servían para la observación del uso de las vocales susurradas. 
4.4. Variación diafásica y observaciones complementarias

La variación diafásica no se incluía entre los objetivos principales del presente estudio, pero en los resultados de las entrevistas encontramos algunos datos interesantes al respecto. Se trata de contexto, cuando el participante repetía la misma palabra varias veces. La primera vez se pronunciaba de manera espontánea, y cuando el entrevistador fingía no comprender bien, la repetía de manera más cuidadosa. El resultado previsto de este experimento era recibir respuestas sin vocal susurrada en la primera respuesta y con la vocal susurrada cuando el participante prestaba más atención, por considerar esta forma más correcta, más vernácula. El experimento se realizó solo en algunas entrevistas dependiendo de la situación. Otras veces los participantes repetían la palabra de manera espontánea, sin ningún estímulo. Las observaciones no confirman la hipótesis de una manera convincente. En general, encontramos participantes que repetían las palabras del mismo modo - como es natural-, también encontramos participantes que de verdad pronunciaron la vocal susurrada al pronunciar de manera más cuidadosa. Sin embargo, también había participantes, que pronunciaron la vocal susurrada en la primera pronunciación -más espontánea- mientras que en la segunda pronunciaron la misma palabra sin esta. Este segundo grupo era menos numeroso que el anterior, pero no tanto como para confirmar la hipótesis. Al final, también encontramos personas que repetían varias veces la misma palabra, unas veces con la vocal susurrada y otras veces sin ella. En resumen, las observaciones de la variación diafásica no aportaron un resultado convincente, parece que los hablantes suelen utilizar las vocales susurradas cuando prestan más atención a la pronunciación, pero algunos lo hacen justo al revés. Este resultado se relaciona con el prestigio de las vocales susurradas. Al parecer, algunos hablantes sienten las formas con la vocal susurrada más prestigiosas y por eso las utilizan cuando se concentran más en la forma de hablar, otros las ven como algo "raro" que tal vez no deba utilizarse en la pronunciación correcta. 
Otras observaciones que surgieron del análisis de los datos se refieren a diferencias entre las palabras individuales. En las tablas 4, 5 y 6 notamos que la frecuencia de aparición de una vocal susurrada varía en función de las palabras estudiadas. Los 84 participantes utilizaron la vocal susurrada en la palabra kaap ${ }^{i}$ en 40 casos, mientras que en la palabra ipiak $k^{u}$ solo en 15 casos. Esto nos lleva a la conclusión que la desaparición de las vocales susurradas no avanza en todo el léxico con la misma rapidez. Es evidente que la palabra kaap ${ }^{i}$ parece conservar más que las demás la pronunciación con la vocal susurrada. Una de las razones se puede deber al valor distintivo que tiene la vocal susurrada en el par kaap ${ }^{i}$ - kaap ${ }^{e}$. No obstante, solo 7 hablantes optaron por distinguir este par por medio de las vocales susurradas, es justo admitir entonces que la relevancia fonológica de las vocales susurradas es mínima. La conservación de la vocal susurrada en la palabra $k a a p^{i}$ se puede deber también a la relación con la cultura material de los shuar. Mientras que el uso del achiote (ipiak ${ }^{u}$ ) para pintarse la cara ya es una realidad pasada, los shuar siguen utilizando la liana kaap ${ }^{i}$ para fabricar las changuinas. En los resultados notamos, además, que la frecuencia de uso depende de la edad del hablante. En la palabra kaap ${ }^{i}$ nos encontramos con frecuencia de uso casi igual en las tres generaciones (12 - 15 - 13), mientras que la palabra ipiak ${ }^{u}$ pronuncia con la vocal susurrada sobre todo la generación más mayor (0 - 3 - 12). Las razones por las cuales la desaparición de las vocales susurradas no avanza de manera igual en todo el léxico son difíciles de descubrir y verificar, pero fenómenos parecidos se han llegado a observar también en otras situaciones de contacto (Valeš 2005).

A pesar de que las vocales susurradas han desaparecido del habla de muchos hablantes, sus realizaciones plenas — cuando se añade un sufijo a la palabra - siguen siendo parte íntegra del habla de todos. De esta manera, hasta los hablantes que pronuncian [ipják] dicen [ipjákuitii], sabiendo que hay una u "omitida".

Como se menciona en la parte metodológica, la recogida de datos incluía también narración libre, sin embargo, en esta parte de la entrevista no solían aparecer las vocales susurradas, ya que estas solo se realizan al final del enunciado. En cambio, la observación 
participante en las comunidades descubrió el uso de las vocales susurradas, ya que los hablantes las usaban cuando pronunciaban palabras sueltas. Estas observaciones, sin embargo, son difíciles de cuantificar con respecto a las tres variables estudiadas. La impresión del investigador adscribe su mayor ocurrencia a las comunidades de la localidad Macuma, no obstante, se trata de una observación subjetiva que puede ser influida por muchos factores.

\section{Conclusión}

El estudio de las vocales susurradas observó la variación de la lengua shuar y confirmó la gradual desaparición de estos fonemas. El uso de las vocales susurradas en los discursos espontáneos era muy poco frecuente, hasta por parte de las personas que los utilizaron respondiendo a los estímulos, y solo se ha conseguido documentarlo de una forma excepcional. Por lo que se refiere a las entrevistas dirigidas, aparecieron más casos de uso de las vocales susurradas de lo que estaba previsto. De los 84 participantes 46 (55\%) utilizaron una vocal susurrada por lo menos una vez. En términos generales, las vocales susurradas todavía siguen estando presentes en la lengua, ya que el número total de sus empleos en todas las entrevistas era 82, eso significa que no se trata solo de usos residuales. El uso de las vocales susurradas en el habla espontánea fue comprobada también a través de la observación participante. No obstante, de igual forma se comprobó que las vocales susurradas están en proceso de gradual desaparición de la lengua, ya que hubo muchos participantes que no utilizaron ni una sola forma de estas y otros que los utilizaron en una o dos palabras de las seis.

Respecto a la variación diastrática y diatópica, se consideraban las variables edad, localidad y grado del bilingüismo con la hipótesis de encontrar una fuerte estratificación por la variable edad. No obstante, los resultados confirman esta hipótesis solo parcialmente. Considerando las cifras absolutas, las generaciones más mayores efectivamente superan a la más joven con el doble de usos, pero, por otro lado, la cantidad de personas que utilizaron 
la vocal susurrada por lo menos una vez es casi igual en las tres generaciones, además, se registró el uso de las vocales susurradas por hablantes muy jóvenes. En cambio, la variable localidad aportó resultados mucho más convincentes. En las dos localidades con el contacto más intensivo y prolongado con la lengua española — valle del Upano y Gualaquiza- encontramos usos excepcionales de las vocales susurradas, solo 6 participantes y 12 usos en total. Al contrario, en las comunidades de Transcutucú los usos de los fonemas estudiados eran frecuentes, las utilizaron $70 \%$ de los participantes. Esta situación indica mayor influencia de la variable localidad en comparación con la edad. La variable grado del bilingüismo no solo refleja la habilidad del individuo de comunicar en ambos idiomas, sino que también parcialmente proyecta las dos variables anteriores. Las personas mayores estaban con pocas excepciones más competentes en shuar y al mismo tiempo las personas de Transcutucú tenían más capacidad de hablar shuar. Por esta razón, el único resultado sorprendente fue el bajo uso de las vocales susurradas por parte de las personas monolingües en shuar. Sin embargo, como se ha explicado anteriormente, este resultado tiene que ver con la metodología de las entrevistas y no con la desaparición de las vocales susurradas del habla de estas personas. En resumen, sería equivocado afirmar que la desaparición gradual de las vocales susurradas se debe solo a uno de los factores estudiados, cada uno de los tres juega su papel. No obstante, el que más influencia demostró fue el factor localidad, que refleja el contacto con la lengua española y la cultura mestiza. De los datos disponibles parece que, en las localidades de más contacto con el español, hasta las personas de más edad y plenamente bilingües dejan de usar las vocales susurradas. Este resultado sugiere, a la vez, que la gradual desaparición de las vocales susurradas, avanza de manera acelerada por la influencia de contacto lingüístico.

Respecto del prestigio de las vocales susurradas, observado por la variación diafásica, no llegamos a resultados unívocos. Algunos hablantes demostraban la tendencia de utilizarlas en la pronunciación cuidadosa, pero también encontramos individuos 
con tendencia contraria. En cambio, se ha comprobado que la desaparición de las vocales susurradas no avanza con igual ritmo en todo el léxico. En la palabra kaap ${ }^{i}$ se registraron 40 usos mientras que en las demás el máximo fue de 15 usos.

En las situaciones de contacto lingüístico no es nada excepcional que los sistemas de las lenguas minoritarias se ajusten respecto a los modos y puntos de articulación a las lenguas de gran dominio, es decir, que el sonido que no corresponde a ninguno en la lengua dominante se substituye por un sonido "parecido" que comparte algunos rasgos con el sonido original. En el caso de las vocales susurradas shuar, observamos una situación diferente, en lugar de su sustitución por una vocal plena correspondiente, la lengua ha optado por su eliminación. Esta eliminación avanza gradualmente por influencia de la situación de contacto. Los datos del análisis demostraron que no se trata de un proceso natural de evolución de la lengua - no influye solo la variable edad-, sino que el contacto con la lengua española es uno de los factores decisivos en el proceso de la pérdida de las vocales susurradas. De este modo, la lengua española influye notablemente en la evolución precipitada de la lengua shuar.

\section{Referencias bibliográficas}

Bolla, Luis

1972 Diccionario práctico del idioma shuar. Quito, Sucúa: Vicariato de Méndez.

BotTASSO, Juan

1982 Los shuar y las misiones: Entre la bostilidad y el diálogo. Quito: Abya-Yala.

1993 Los salesianos y la Amazonía, volumen 3. Quito: Abya-Yala.

2003 Los salesianos y la lengua de los shuar. Quito: Abya-Yala.

2011 Los salesianos y los shuar. Quito: Abya-Yala.

BROSEGHINI, Silvio

1983 Cuatro siglos de misiones entre los shuar. Sucúa: Mundo shuar.

Constitución de la República del Ecuador

s.f. Texto completo disponible en: $<$ http://biblioteca.espe.edu.ec/ upload/2008_2.pdf>. 
FAST MowiTz, Gerhard

1975a Descripción comparativa de los rasgos distintivos fonémicos de varios idiomas de la familia lingüistica jíbara. Lima: Ministerio de Educación, ILV - DEL - 21.

1975b Análisis tentativo del sistema fonológico del idioma jíbaro del río corrientes. Lima: Ministerio de Educación, ILV - DEL - 21.

GHINASSI, Juan

1939 Vocabulario. S/d. Quito.

GNerre, Maurizio

1999 Profilo descriptivo e storico-comparativo di una lengua amazónica: lo shuar (Jívaro). Nápoles: Universitá di Napoli 'L'Orientale'.

2010 Perfil descriptivo e bistórico-comparativo de una lengua amazónica: el shuar (Jíbaro). Bilbao: Servicio Editorial de la Universidad del País Vasco.

2012 "Los salesianos y los shuar construyendo la identidad cultural". En La presencia Salesiana en el Ecuador: Perspectivas históricas y sociales. Coords., Lola Vázquez et al. Quito: AbyaYala, 573-635.

HANZelka, Jiř́ y Miroslav Zikmund

1958 Za lovci lebek. Praha: Orbis.

Harner, Michael

1984 The Jivaro. People of the Sacred Waterfalls. Berkeley, Los Angeles: University of California Press.

Henriksen, Luis A. y Pedro V. Obando

1985 Mane pinkìb kamshimtus: Gramática pedagógico-práctica de cuaiquer. Pasto, Nariño: Universidad de Nariño.

KARSTEN, Rafael

1935 The Head Hunters of Western Amazonas. Helisingfors: Societas Scientiarum Fennica, Commentationes Humanarum Litterarum, VII.1.

1989 La vida y la cultura de los shuar, volumen 2. Quito: AbyaYala.

LABOv, William

1972 Sociolinguistic Patterns. Philadelphia: University of Pennsylvania Press. 
Matras, Yaron

2009 Language contact. Cambridge: Cambridge University Press.

[Pellizzaro] Pellizaro, Siro y Fausto Oswaldo Náwech

2005 Chicham. Diccionario shuar-castellano. Quito. Abya Yala.

SERBISH

2004 Chicham nekatai. Diccionario comprensivo castellano-shuar. Sucúa: Sistema de educación radiofónica bicultural shuar.

SiguÁn, Miguel

2001 Bilingüismo y lenguas en contacto. Madrid: Alianza Editorial.

STIRLING, Matthew Williams

1938 "Historical and Ethnographical Material on the Jibaro Indians". Buereau of American Etbnology Bulletin. Washington D. C., $117,1-148$.

Turner, Glen D.

1958a Jivaro Phonology and Morphology. Unpublished doctoral dissertation. Bloomington: Indiana University.

1958b "Alternative Phonemicizing in Jivaro". International Journal of American Linguistics. 24, 2, 87-94.

1992 "Una breve gramática del shuar". Cuadernos Etnolingüísticos. Quito, 19.

VALEŠ, Miroslav

2005 "Aspectos léxicos del contacto entre shuar y español". IberoAmericana Pragensia. 39, 199-205.

2011 "Vitalidad de la lengua shuar y actitud de los hablantes hacia su lengua vernácula". En Endangered Languages: Voices and Images. Eds., Marleen Haboud y Nicholas Ostler. Bath: Foundation for Endangered Languages, 68-72.

s/a El sistema vocálico shuar: vocales susurradas, nasales y largas (en revisión).

WeINREICH, Uriel

1979 Languages in Contact. The Hague: Mouton Publishers.

Wipio Deicat, Gerardo

1996 Diccionario aguaruna-castellano, castellano-aguaruna. Lima: Instituto Lingüístico de Verano, Serie Lingüística Peruana no. 39.

Fecha de recepción: 1/04/2015

Fecha de aceptación: 19/12/2016 